\title{
Contour separation and orientation in shape discrimination by rats '
}

\author{
P. C. DODWELL AND R. R. NIEMI \\ QUEEN'S UNIVERSITY, KINGSTON, ONTARIO
}

Several theoretical models to account for shape discrimination by rats have been proposed. A central issue has been the relative importance of contour separation or contour orientation as a major factor in the coding system. The models of Deutsch and Dodwell reflect this disagreement, Deutsch favoring the former variable and Dodwell the latter. Three stimulus figures were devised in such a way that two of them had equal and constant contour separation, and two had equal amounts of contour in the horizontal and vertical orientations. It was found that the pair with identical contour separation were readily discriminable from one another, but the pair with identical orientations were not. It was concluded that contour orientation is more important in the rat's pattern coding system than contour separation.

A number of attempts have recently been made to account for shape recognition in such organisms as the rat and octopus (Deutsch, 1955, 1962; Dodwell, 1957. 1964; Sutherland, 1957, 1959). These attempts make the common assumption that spatial information on the retina is coded before recognition occurs, but differ in their proposals about the basis of coding. Deutsch has consistently employed the notion that a shape-coding system be based on the computation of intercontour distances, and cites anatomical evidence for the octopus and bee in favor of it. Sutherland's original model (1957), although based on separation of horizontal and vertical components in a pattern, also employs a system which computes, basically, intercontour distances. Dodwell (1957), on the other hand, has used the principle of coding by the extent of contours in different orientations-particularly horizontal and vertical-and subsequently cited supporting neurophysiological evidence from the visual system of the cat (Dodwell, 1964). There is no compelling reason to suppose that shape-coding systems in mammals are the same as in submammalian vertebrates and invertebrates; indeed there are anatomical and physiological grounds for expecting them to be different. Be this as it may, it seemed worthwhile to find out whether both contour separation and contour orientation have importance in shape recognition in the rat. The experiment reported here is also a test between the coding systems of Deutsch and Dodwell, although the oversimplifications inherent in both models are recognized.

The stimuli used are shown in Fig. 1. These patterns satisfy several criteria for a test of a shaperecognition system per se. That is, they have equal brightness, the same general orientation, and bilateral symmetry. Other factors may contaminate a test for

properties of a shape-recognition system, e.g., difference in size (Fields, 1931) and the relation of figure edges to apparatus boundaries (Dodwell, 1957; Sutherland, 1961); however, these can be controlled by presenting white outline stimuli varying in size from trial to trial in a darkened apparatus, and by various part-figure tests.

The shapes $A$ and $\mathrm{C}$ of Fig. 1 display contour orientation uniformity, but contour separation disparity. A and B display orientation disparity but separation uniformity. Although space precludes an exact derivation, it can be shown that for Deutsch's model $A=B \neq C$, and for Dodwell's, $A=C \neq B$. The experiment tests these predictions.

Method

Ss were eight male hooded rats approximately three months old, maintained at $80 \%$ normal body weight, and handled for $1 / 2 \mathrm{~h}$ per day for seven days prior to experimentation. Ss were randomly assigned to two equal groups.

The apparatus consisted of a blackened, narrow chamber, $12 \times 4 \times 4$ in. high. One end widened to 6 in. to contain two clear plastic panels 2 in. square and set 2 in. apart. Small cups delivering a standard food reinforcement pellet were located immediately beneath each panel. The other end of the chamber contained a horizontal bar.

Stimuli were white outline pairs of A and B or A and $C$ on a black ground, mounted on slides and projected onto a ground glass screen behind the panels, one shape appearing behind each panel, in a Gellerman series. (The shapes provided the only illumination in the apparatus.) Sixteen pairs of stimuli were used, size varying by $24 \%$. At the maximum viewing distance the contour width subtended $0.25^{\circ}$, i.e., well above the hooded rat's acuity threshold.

After shaping, the trial sequence was as follows. A bar press at the back of the box caused stimulus onset. A correct panel response (press) effected offset of the negative shape and presentation of food during $5 \mathrm{sec}$ continued illumination of the positive shape. An incorrect response caused immediate off-

A B

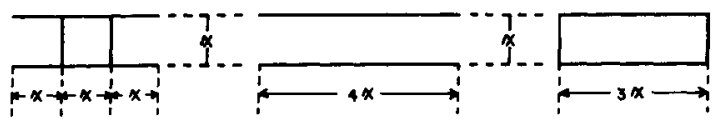

Fig. 1. Stimuli used in the experiment, shown in algebraic proportions. In the study, they were white outline figures on a black ground. 


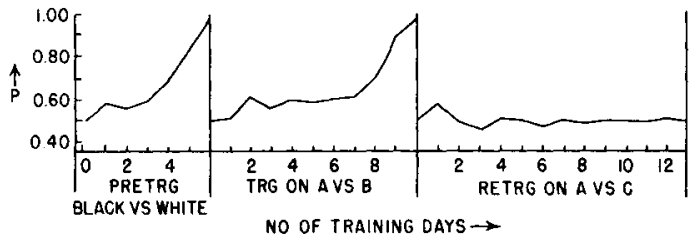

Fig. 2. P, mean proportion correct responses per block of $\mathbf{4 0}$ training trials vs days of training ( 40 trials per day) for Group 1.

set of both shapes. The intertrial interval was 5 or $30 \mathrm{sec}$, for correct and incorrect responses, respectively.

After pretraining on a black-white discrimination, Group 1 was trained on $A$ and $B$, then on $A$ and $C$, 40 trials per day. For Group 2 the order was reversed.

Where learning occurred $(80 \%$ correct responses per day or better), transfer tests for part-figure, brightness and size discrimination were given. Where learning did not occur (400 trials without statistically observable effects), reversal of reinforcement value of the stimuli was tried, but had no observable effects. Retraining on a black-white discrimination was then instituted for non-learners. Reinforcement values of stimuli were appropriately counterbalanced. (Since part-figure etc. tests, and reinforcement counterbalancing etc. had no observable effects, these data are not reported.)

\section{Results}

Results are shown in Figs. 2 and 3 above. No statistical tests of the reliability of the choice between $A$ and $B$ was deemed necessary, since all Ss reached the (stringent) criterion at Day 10 or better. No $S$ displayed better than chance performance on $A$ and C. No $S$ showed reliable preference in the part-figure transfer tests (top halves, bottom halves), thus indicating that they were responding to the whole patterns. Discussion

The findings lend strong support to the hypothesis that contour orientation plays an important role in rats' ability to discriminate shape. It also appears that contour separation, at least in this situation, is a much less effective cue, although it is not possible to prove that it is always entirely ineffective.

The test made is deemed unequivocal for the following reasons. First, confounding discriminatory factors such as brightness, size and general figure orientation were controlled, and appropriate transfer tests yielded

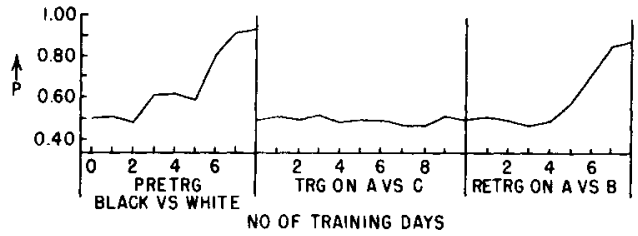

Fig. 3. P, mean proportion correct responses per block of 40 training trials vs days of training ( 40 trials per day) for Group 2 .

no evidence suggesting that discrimination was based on part-figures, or other similar factors. Secondly, rats could discriminate $A$ and $B$, but not $A$ and $C$, irrespective of training order, although they displayed identical behavior in the two situations in other respects (thus "failure of attention" is ruled out).

Although work to date favors a contour orientation hypothesis in discrimination of shape by rats, neither this hypothesis nor the Dodwell model is supported to the exclusion of other possibilities. However, a surprising degree of convergence with the neurophysiological findings (e.g., Hubel \& Wiesel, 1962) is evident, as in other situations (Dodwell, 1965).

\section{References}

DEUTSCH, J. A. A theory of shape recognition. Brit. J. Psychol, 1955 , 46, 30-37.

DEUTSCH, J. A. A system for shape recognition. Psychol Rev., 1962 , $69,492-500$.

DODWELL, P. C. Shape recognition in rats. Brit. J. Psychol., 1957, 48, 221-229.

DODWELL, P. C. A coupled system for coding and learning in shape discrimination. Psychol. Rev., 1964, 71, 148-159.

DODWELL, P. C. Anomalous transfer effects after shape discrimination training in the rat. Psychon. Sci., 1965, 3, 97-98.

FIELDS, P. E. Contributions to visual figure discrimination in the white rat. J. comp. physiol. Psychol, 1931, 11, 349-367.

HUBEL, D. H., \& WIESEL, T. N. Receptive fields, binocular interaction and functional architecture in the cat's visual cortex.J. Physiol., 1962, $160,106-154$.

LASHLEY, K. S. The mechanism of vision: XV. Preliminary studies of the rat's capacity for visual detail. J. gen. Psychol., 1938, 18, 123-193.

SUTHERLAND, N. S. Visual discrimination of orientation by octopus. Brit. J. Psychol, 1957, 48, 55-71.

SUTHERLAND, N. S. A test of a theory of shape discrimination in Octopus Vulgaris Lamarck. J. comp. physiol. Psychol., 1959, 52, 135-141.

SUTHERLAND, N. S. Shape discrimination by animals. E. P. S. Monogr. No. $1,1961$.

Note

1. This research was supported by grant APT 109 of the National Research Council of Canada to the first author. 